\title{
Linear and Non-linear Stability of Heat Exchangers
}

\author{
A. C. Fowler $\dagger$ \\ Mathematical Institute, Oxford, England
}

[Received 16 June 1977 and accepted 10 April 1978]

\begin{abstract}
The hydrodynamical problem of one-dimensional flow with a uniform heat input resulting in a change of phase is considered. Equations of mass, momentum, energy and state representing the dynamic behaviour of such a system are reduced to two coupled equations for the density $\rho(x, t)$ and the inlet velocity $U(t)$ on the assumption that the pressure drop applied between the inlet and the outlet is "small". A linear stability analysis is carried out which leads to the problem of computing the zeros of a complicated analytic function. A non-linear analysis is applied to the case of weak instability to find the evolution of the slowly varying amplitude of a small oscillation: in certain circumstances, a "burst" occurs, and in such cases no such small oscillation can exist.
\end{abstract}

\section{Introduction}

A PROBLEM OF much interest to industrial engineers is that of determining the stability properties of two-phase fluid flow in boilers and nuclear reactors. A typical situation is when the flow is driven by an applied pressure drop through a bank of parallel channels, as shown in Fig. 1. The fluid, in its liquid phase at the inlet, is subjected to a strong external heat in put so that it undergoes a change of phase as it proceeds up the channels. If the pressure drop and heat input are given, then the system shown in Fig. 1 is self-contained, and one is then interested in determining steady-state values $u_{w}$ of the inlet velocity, and the stability of these to small perturbations of the system.

There are two possible approaches we may adopt. In view of the complexity of the system (the flow is turbulent and comprises two phases), one may attempt to construct as realistic a model as possible, and thus hope to gain a reasonable comparison with experimental results: such models require numerical work from the outset, however, and offer little hope of analytical results; alternatively, we may choose a simpler model which it is hoped will reflect the qualitative behaviour of the system, and which is capable of some analysis. It is this second approach which we adopt in this paper.

The model that we shall use is based on the models of earlier authors (e.g. Zuber, 1967; Davies \& Potter, 1967) who also studied the linear stability of steady-state solutions. In this sense, the linear stability of flow through a single boiling channel may be said to be well understood. We find that the equations can be partially solved,

$\dagger$ Present address: School of Mathematics, Trinity College, Dublin 2, Eire.

$0020-2932 / 78 / 070361+22 \quad 502.00 / 0$ 
so that the original model is reformulated as a pair of delay integro-differential equations for the (dimensionless) density $\rho(x, t)$ and inlet velocity $U(t)$ : this is done in Section 2. The method is slightly different from that of earlier authors (e.g. Davies $\&$ Potter, 1967) in that the equations are simplified before the stability is examined by linearization.

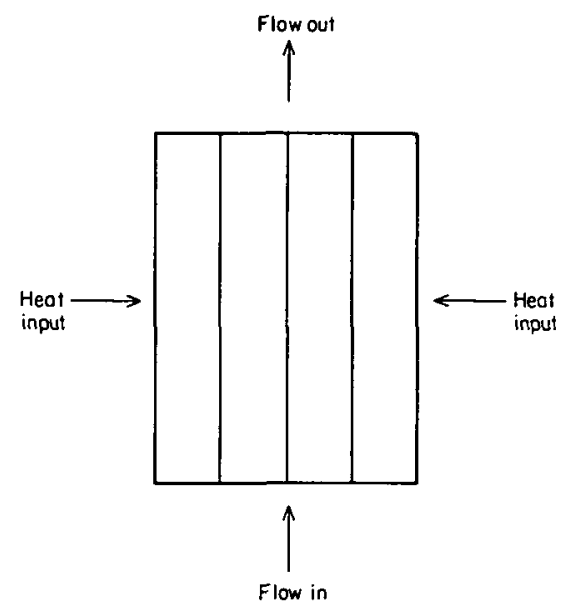

FIG. 1. A once-through heat exchanger.

In Section 3, we examine the steady-state solutions (there may be more than one) and assess their linear stability. This is in essence a recasting of previous work. In passing, we prove Ledinegg's (1938) result that negative-slope regions on the equilibrium pressure drop/flow curve are associated with monotonic instabilities, which seems previously to have been supported only by dubious physical arguments. The converse, however, is not generally true (as is confirmed in Section 5, where a numerical counter-example is given).

In Section 4 we turn to the principal purpose of the present paper, a non-linear analysis of a small amplitude oscillation by the method of multiple scales, as described by Stuart (1960). The only previous work in this area appears to be that of Friedly \& Krishnan (1974), who used Poincaré's method to compute the amplitude of a small, steady oscillation when the equilibrium of the system has a slight oscillatory instability. The work does not describe how this limiting solution is approached, or what happens if no such solution exists. In Section 4 we show how the method of multiple scales may be used in the present problem to obtain an evolution equation for the amplitude of an oscillatory disturbance from the steady state. There are then two choices: firstly, that a limiting amplitude is indeed reached; secondly, that a "burst" occurs, that is, the amplitude becomes infinite in a finite time. The asymptotic solution then becomes invalid, and the steady state may be said to be non-linearly unstable; in this case little further can be said analytically. However, it is reasonable to suppose that a "burst" is associated with large amplitude oscillatory behaviour, and thus the system may exhibit a kind of "threshold excitation" between 
large and small oscillations in certain parameter ranges. Such a phenomenon appears to have been experimentally observed by Jain (1965).

In Section 5 we give the results of some numerical computations on the analytic results of previous sections, and these results are discussed in Section 6.

\section{Mathematical Model}

Let us consider a heated one-dimensional flow in a pipe $0<x<l$, which reaches its saturation temperature at a point called the boiling boundary. For simplicity we shall suppose that the outlet is reached before the two-phase mixture becomes dry steam, so that the flow consists of two regions only. (This assumption is not necessary to the analysis.) The flow is subject to a constant heat input $Q$ per unit length per time and is supposed driven by a constant pressure drop $\Delta p$.

With the assumption that the two-phase region is homogeneous, suitable equations of motion are

$$
\begin{gathered}
\rho_{t}+u \rho_{x}+\rho u_{x}=0, \\
p_{x}=-\kappa \rho u^{2}, \\
\rho \frac{d e}{d t}+p u_{x}=Q / A,
\end{gathered}
$$

where $\rho, u, p, e$ are the density, velocity, pressure and specific internal energy respectively, $A$ is the cross-section of the tube, and $\kappa$ is a given constant. Suffixes denote partial derivatives and $d / d t$ is the total (material) derivative. The term $\kappa \rho u^{2}$ in (2.2) is an empirical friction force used to model the turbulence of the flow (Davies \& Potter, 1967). We have neglected the frictional dissipation in the energy equation (2.3) and the inertial terms in the momentum equation (2.2). This latter approximation is valid if $\kappa$ is "large" enough (more specifically if $\beta$ in (2.20) is large enough), but we wish to emphasize that this simplification is made purely to shorten the arithmetic (which is in any case considerable), and is not necessarily intended to be a realistic approximation. In a particular application, it would be advisable to incorporate inertial terms, but their inclusion does not invalidate the method described in Section 4.

As equation of state, we assume that the water is incompressible so that

$$
\frac{d \rho}{d t}=0
$$

there. In the two-phase region, the usual Clausius-Clapeyron relation between pressure and saturation temperature is given by

$$
\frac{\delta p}{\delta T}=\frac{L}{T v_{f g}},
$$

where $\delta T$ is the change in the saturation temperature $T$ corresponding to a change $\delta p$ in the ambient pressure $p, L$ is the latent heat, and $v_{f g}$ is the change in specific volume between liquid and vapour. If we assume 


$$
\frac{v_{g g} \delta p}{L} \ll 1
$$

then also

$$
\frac{\delta T}{T} \ll 1
$$

and we may realistically suppose that the saturation temperature $T$ is effectively constant. In this case the densities and specific internal energies of both steam and water phases, $\rho_{s}, e_{s}, \rho_{w}, e_{w}$ are constant, as is the latent heat $L$. (These formal assumptions are found to be accurate when checked against steam tables.) The assumption (2.6) is valid if the pressure drop $\Delta p$ along the tube is sufficiently small. Since $v_{f g}=1 / \rho_{s}-1 / \rho_{w}$, and $\rho_{s}<\rho_{w},(2.6)$ is certainly valid provided

$$
\frac{\Delta p}{\rho_{s} L} \ll 1 \text {, }
$$

and this condition is usually satisfied in applications. For instance, if $\Delta p=1$ bar at an ambient pressure of $\sim 30$ bars, then $\Delta p / \rho_{s} L \sim 3 \times 10^{-3}$. We therefore take the abovedefined thermodynamic variables to be constant. In particular, the latent heat $L$ is defined by

$$
\begin{aligned}
L & =e_{s}-e_{w}+p / \rho_{s}-p / \rho_{w}, \\
& =e_{s}-e_{w}+p_{\text {out }}\left(\frac{1}{\rho_{s}}-\frac{1}{\rho_{w}}\right)+\dot{O}\left(\frac{\Delta p}{\rho_{s} L}\right) L,
\end{aligned}
$$

so that dividing by $\rho_{w} L\left(1 / \rho_{s}-1 / \rho_{w}\right)$, we obtain

$$
\left(\frac{\rho_{w}}{\rho_{s}}-1\right)^{-1}=\frac{1}{\rho_{w} L}\left[\frac{\rho_{w} \rho_{s}\left(e_{s}-e_{w}\right)}{\rho_{w}-\rho_{s}}+p_{\text {out }}\right]+O\left(\frac{\Delta p}{\rho_{w} L}\right)
$$

This result is used below in (2.22). Additionally, in view of (2.4) above, $\rho_{w}$ being constant requires that we specify $\rho=\rho_{w}$ at $x=0$, as in (2.18) below. With these assumptions, addibility of mass and internal energy in the two-phase region imply that

$$
\rho=\rho_{w}(1-\alpha)+\rho_{s} \alpha
$$

and

$$
\rho e=\rho_{w} e_{w}(1-\alpha)+\rho_{s} e_{s} \alpha
$$

there, where $\alpha$ is the void fraction (fraction by volume of steam), and (2.10) and (2.11) simultaneously define the equation of state for the two-phase mixture.

Eliminating $\alpha$ between (2.10) and (2.11), we obtain

$$
e=\beta_{1} / \rho+\beta_{2}
$$

where

$$
\beta_{1}=\frac{\rho_{w} \rho_{s}\left(e_{s}-e_{w}\right)}{\rho_{w}-\rho_{s}}, \quad \beta_{2}=\frac{\rho_{w} \rho_{w}-\rho_{s} e_{s}}{\rho_{w}-\rho_{s}}
$$


From (2.1) and (2.12), we find

$$
\rho \frac{d e}{d t}=\frac{-\beta_{1}}{\rho} \frac{d \rho}{d t}=\beta_{1} u_{x},
$$

and so in the two-phase region (2.3) becomes

$$
\left[\beta_{1}+p\right] u_{x}=Q / A \text {. }
$$

If we define the boiling boundary as $r(t)$, so that

$$
e=e_{w} \text { on } x=r(t)
$$

then (2.15) is valid in $r(t)<x<1$, and (2.4) is valid in $0<x<r(t)$. In order that $x=1$ is reached before the flow becomes one of pure steam, we require

$$
\alpha<1 \text { on } x=l \text {. }
$$

In this case the boundary conditions for the equations are that

$$
\begin{aligned}
& e=e_{0}, \quad \rho=\rho_{w} \text { on } x=0, \\
& u, \rho \text { are continuous across } x=r(t), \\
& \Delta p=\text { constant (given). }
\end{aligned}
$$

Here, $e_{0}$ and $\rho_{w}$ are given constants. We show below that these conditions are sufficient to determine the solution.

In order to proceed further, we non-dimensionalize the equations. Let $u_{w}$ be a steady-state value (as yet unknown) of the inlet velocity (there may be more than one corresponding to a given pressure drop). We define dimensionless variables by writing

$$
\begin{aligned}
\rho & =\rho_{w} \bar{\rho}, \quad u=u_{w} \bar{u}, \quad x=\frac{\rho_{w} u_{w} L A}{Q} \bar{x}, \\
e & =L \bar{e}, \quad \dot{t}=\frac{\rho_{w} L A}{Q} \bar{t}, \quad p=p_{\mathrm{out}}+(\Delta p) \bar{p}, \\
r(t) & =\frac{\rho_{w} u_{w} L A}{Q} \dot{R}(t) .
\end{aligned}
$$

Here $L$ is the latent heat and $p_{\text {out }}$ is the outlet pressure. The choice of $x$ scaling is motivated by the fact that in the steady state $\rho_{w} u_{w} A$ is the (constant) mass flow rate, and so (with $L$ constant), $\rho_{w} u_{w} L A / Q$ would simply be the length of the two-phase region, if the tube were so long that $\alpha$ reached 1 before $x=l$. This is clearly the natural length scale of the problem.

On using (2.19), and dropping the bars on these dimensionless variables for convenience, the two-phase equations (2.1), (2.2) and (2.15) become

$$
\begin{aligned}
\rho_{t}+u \rho_{x}+\rho \dot{u}_{x} & =0, \\
p_{x} & =-\beta \rho u^{2}, \\
(\sigma \omega p+1 / \mu) u_{x} & =1,
\end{aligned}
$$


where

$$
\begin{aligned}
\beta & =\frac{\kappa \rho_{w}^{2} u_{w}^{3} L A}{Q(\Delta p)}, \quad \omega=\rho_{s} / \rho_{w}, \quad \sigma=\Delta p / \rho_{s} L, \\
1 / \mu & =\left(\beta_{1}+p_{\text {out }}\right) / \rho_{w} L .
\end{aligned}
$$

From the definition of $\beta_{1}$ in (2.13) and using (2.9), we see that (neglecting $O\left(\Delta p / \rho_{s} L\right)$ )

$$
\mu=\frac{1-\omega}{\omega} \text {. }
$$

Since by choice of scaling in (2.19) $p \sim 1$, from (2.22) $\mu \omega \sim 1$, and by assumption $\sigma \ll 1$ in $(2.21)$, it follows that

$$
\sigma \mu \omega p \sim \sigma \ll 1
$$

in (2.20), i.e. $\sigma \omega p$ is much smaller than $1 / \mu$. We make this statement formally precise by considering the asymptotic limit

$$
\sigma \rightarrow 0, \quad \mu, \beta, \omega=O(1) \text {, i.e. finite. }
$$

A slight qualification must be made to (2.24). At low operating pressures, the ratio $\omega$ is quite small; for example $\omega \sim 1 / 50$ at 33 bars: hence also $\mu \sim 50$. Furthermore, neglecting $\sigma$ in (2.20), we have $u_{x}=\mu$, and since $\rho u=1$ in the steady state, it follows from the second equation in (2.20) that $p \sim \beta / 2 \mu \omega^{2}$, since $u \rightarrow 1 / \omega$ as $\alpha \rightarrow 1$ (the fact that we constrain $\alpha$ to be less than one is irrelevant here); by definition, $p \sim 1$, also $\mu \omega \sim 1$, hence $\beta \sim \omega$. One might suppose, therefore, that there is some kind of limiting process associated with $\omega \rightarrow 0$; however, this is not so, and such an asymptotic analysis would be irrelevant. In view of this, we formally consider $\omega$ as $O(1)$, although in reality it may be quite small. In any case, it is clear from (2.23) that the limit (2.24) accurately represents the model.

Putting $\sigma=0$ in (2.20), we find that the energy equation in the two-phase region reduces to the simple form

$$
u_{x}=\mu \text {. }
$$

(A similar equation may be derived in a zone of dry steam, if this is assumed to obey the perfect gas law: the derivation is much the same.)

Let $U(t)$ be the (unknown) dimensionless inlet velocity, so that $U=1$ is a steady solution of the problem (see Section 3 below). Since the water is incompressible, we have $u \equiv U(t)$ and $\rho \equiv 1$ in $0<x<R(t)$, hence the dimensionless form of $(2.3)$ is easily seen to be

$$
e_{t}+U(t) e_{x}=1 \text {. }
$$

This equation may be solved for $e$ by the method of characteristics. In particular, we find that the boiling boundary $R(t)$ is given exactly by

$$
R(t)=\int_{t-\tau_{0}}^{t} U(\theta) d \theta,
$$

where the delay $\tau_{0}=\left(e_{w}-e_{0}\right) / L$ is the dimensionless transit time from the inlet to the boiling boundary: it is seen to be proportional to $e_{w}-e_{0}$, which may be taken as a 
measure of the "inlet sub-cooling", i.e. the difference between the inlet temperature and the saturation temperature, and so in general we shall simply refer to $\tau_{0}$ as the inlet sub-cooling. We define the non-dimensional length $\lambda$ of the tube as

$$
\lambda=\frac{l Q}{\rho_{w} u_{w} L A},
$$

and then we can write the equations for $u$ and $\rho$ in the two-phase region from (2.25) and (2.20) as

$$
\begin{aligned}
u_{x} & =\mu, \\
\rho_{\imath}+u \rho_{x}+\rho u_{x} & =0
\end{aligned}
$$

with boundary conditions

$$
u=U(t), \quad \rho=1 \text { on } x=R(t),
$$

where $R(t)$ is defined by (2.27). The final boundary condition in (2.18) is then satisfied, from (2.20), by specifying

$$
\beta \int_{0}^{\lambda} \rho u^{2} d x=1 .
$$

Since $\rho u^{2}=U^{2}(t)$ in $x<R(t)$, we may write $(2.31)$ in the form

$$
U^{2}(t) R(t)+\int_{R(t)}^{\lambda} \rho u^{2} d x=\frac{1}{\beta} .
$$

The system to be solved is now (2.29) and (2.32), with the boundary conditions (2.30) on $R(t)$, given by (2.27). In principle, we also require an initial condition for $\rho$ but this can safely be left arbitrary. The unknowns in the problem are $U(t)$ and $\rho(x, t)$. Assuming $U$ known, we may solve (2.29) and (2.30) to find $u$ and $\rho$ in terms of $U$, and then $U(t)$ is to be determined as the solution of the remaining equation (2.32). The above system of equations remains valid provided $\alpha<1$ at $x=\lambda$ (the case $\lambda<R(t)$ is trivial).

\section{Steady-state and Linear Stability}

The system of equations and boundary conditions (2.29), (2.30) and (2.32) described in Section 2 is sufficient to determine $U(t)$ (and hence $\rho(x, t)$ ) if the righthand side of (2.32) is a known constant. $\Delta p$ is a given constant (as is $\rho_{w}$ ) but we have not specified $u_{w}$ : we now do this.

By the definition of $u_{w}, U=1$ is a steady solution of the equations. Substituting successively into the equations (2.27), (2.29), (2.30) and (2.32), we find that in the steady state

$$
\begin{gathered}
R=\tau_{0}, \\
u=1+\mu\left(x-\tau_{0}\right), \quad x>\tau_{0}, \\
\rho=\frac{1}{1+\mu\left(x-\tau_{0}\right)}, \quad x>\tau_{0},
\end{gathered}
$$


and therefore

i.e.

$$
\tau_{0}+\int_{\tau_{0}}^{\lambda}\left[1+\mu\left(x-\tau_{0}\right)\right] d x=\frac{1}{\beta}
$$

$$
\frac{\Delta p}{u_{w}^{3}} \propto \frac{1}{\beta}=\lambda+\frac{1}{2} \mu\left(\lambda-\tau_{0}\right)^{2},
$$

which defines the right-hand side of (2.32) in terms of the three parameters $\mu, \lambda$ and $\tau_{0}$. (3.4) also serves to determine the steady inlet velocity $u_{w}$ as a function of $\Delta p$ and the other inputs to the problem. Since from the definitions of $\mu, \lambda$ and $\tau_{0}$, we have $\mu, \tau_{0}=$ constant and $1 / \lambda \propto u_{w}$ when only $u_{w}$ is allowed to vary, it follows, using the definition of $\beta$ in (2.21), that (3.4) can be written

$$
\Delta p=A u_{w}+B u_{w}^{2}+C u_{w}^{3},
$$

where $A, B, C$ are functions of the parameters $Q, \rho_{w}$, etc., but are independent of $u_{w}$. A typical example of an equilibrium pressure drop/flow curve given by (3.5) is shown in Fig. 2. One can see in this case that there are three possible steady-state values of $u_{w}$.

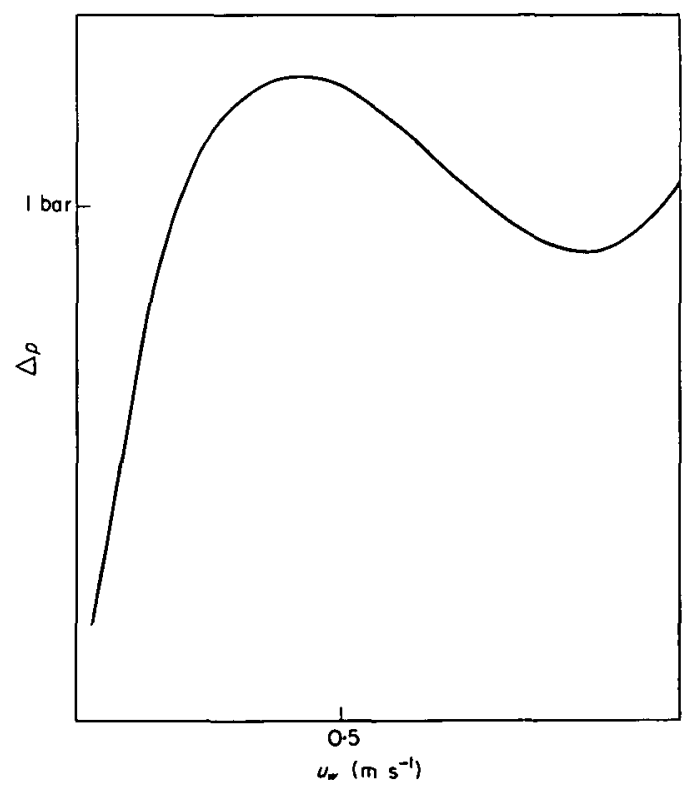

Fig. 2. A typical steady-state pressure drop-flow curve.

Finally, the condition that the outlet should be in the two-phase region may be written, from (2.17), (2.10) and (2.19) as

$$
1>\rho>\omega \text { on } x=\lambda \text {. }
$$


From (3.3) and the definition of $\mu(2.22)$, this is

$$
1<1+\frac{1-\omega}{\omega}\left(\lambda-\tau_{0}\right)<\frac{1}{\omega}
$$

i.e.

$$
0<\lambda-\tau_{0}<1 .
$$

A quantity commonly measured by engineers is the "exit quality", which is the mass fraction of steam at the outlet. From $(2.10)$, this is given by

$$
\frac{\rho_{s} \alpha}{\rho_{w}-\left(\rho_{w}-\rho_{s}\right) \alpha} \text {. }
$$

In the steady state, $\alpha$ is given from (2.10) and (2.19) by

$$
\begin{aligned}
\alpha & =\frac{1-\rho}{1-\omega} \\
& =\frac{1-\left[\frac{1}{1+\mu\left(x-\tau_{0}\right)}\right]}{1-\omega} \\
& =\frac{\mu\left(x-\tau_{0}\right)}{(1-\omega)\left[1+\mu\left(x-\tau_{0}\right)\right]},
\end{aligned}
$$

and so the exit quality is defined by

$$
\frac{\left(x-\tau_{0}\right) /\left[1+\mu\left(x-\tau_{0}\right)\right]}{1-\mu\left(x-\tau_{0}\right) /\left[1+\mu\left(x-\tau_{0}\right)\right]}=x-\tau_{0},
$$

and increases linearly along the tube. (3.7) then simply states that the exit quality be between zero and one, and provides an easy way of measuring the value of $\lambda-\tau_{0}$.

We now collect the time-dependent problem stated in Section 2 as

$$
\begin{gathered}
R(t)=\int_{t-\tau_{0}}^{t} U(\theta) d \theta, \\
u=U(t)+\mu[(x-R(t)], \\
\rho_{t}+u \rho_{x}+\rho u_{x}=1, \quad \rho=1 \text { on } x=R(t), \\
u^{2}(t) R(t)+\int_{R(t)}^{\lambda} \rho u^{2} d x=\lambda+\frac{1}{2} \mu\left(\lambda-\tau_{0}\right)^{2},
\end{gathered}
$$

where we have integrated $u$ using (2.29) and (2.30).

A steady solution of $(3.10)-(3.13)$ is

$$
\begin{aligned}
& U=1, \\
& \rho=\left[1+\mu\left(x-\tau_{0}\right)\right]^{-1} .
\end{aligned}
$$

To examine its linear stability, we put

$$
\begin{aligned}
& U=1+\varepsilon e^{n t}, \\
& \rho=e^{-\mu \xi}+\varepsilon \rho_{0}(\xi) e^{n t},
\end{aligned}
$$


where $\varepsilon \ll 1, \xi=\mu^{-1} \log \left[1+\mu\left(x-\tau_{0}\right)\right]$ and $n$ is in general complex. We neglect $O\left(\varepsilon^{2}\right)$ and solve (3.12) for $\rho_{0}(\xi)$ : substituting this into (3.13), we find that the perturbation of the dimensional pressure drop may be written as

where

$$
\Delta p_{1}=\beta(\Delta p) f(n) e^{n t},
$$

$$
\begin{gathered}
f(n)=2 \lambda+\frac{a}{2 \mu-n}\left[e^{(2 \mu-n) \tau_{1}}-1\right]+\frac{b}{\mu}\left(e^{\mu \tau_{1}}-1\right)-\frac{2 \mu}{n}\left(\lambda-\tau_{0}\right)\left(1-e^{-n \tau_{0}}\right), \\
b=\frac{\mu}{n-\mu}\left[1-\frac{\mu}{n}\left(1-e^{-n \tau_{0}}\right)\right], \quad a=\frac{-\mu e^{-n \tau_{0}}}{n-\mu},
\end{gathered}
$$

and $\tau_{1}=\mu^{-1} \log \left[1+\mu\left(\lambda-\tau_{0}\right)\right]$ is the dimensionless transit time through the twophase region. The flow is then linearly unstable if $f(n)$ has any zeros with $\operatorname{Re} n>0$; otherwise it is stable. The details of this solution are given in Appendix 1.

It is simple to prove $f(n)$ has an infinite number of zeros if $\tau_{1} \neq 0$, but only a finite number with $\operatorname{Re} n>0$. (This is because the zeros are isolated and $f(n) \rightarrow 2 \lambda$ as $|n| \rightarrow \infty$, $\operatorname{Re} n \geqslant 0$.) A reasonable bound is easy to obtain, and the roots can be estimated by scanning the complex plane, thus obviating the need to use Nyquist's criterion. By expanding for small $\tau_{1}$, it can be shown that in this case any zero of $f(n)$ must have $\operatorname{Re} n<0$. In other words, the flow is stable for small exit quality (defined above).

Now suppose we have a general dimensional inlet velocity $u_{w} U(t)$ that induces a dimensional pressure drop $\Delta p$. We may write

$$
\Delta p=N\left[u_{w} U(t)\right],
$$

where $N$ is a non-linear operator. If $U=1+\varepsilon e^{n t}$, then by the analysis above leading to (3.16) we can expand $N$ as

$$
N\left[u_{w}\left(1+\varepsilon e^{n t}\right)\right]=N\left[u_{w}\right]+\varepsilon \beta(\Delta p) f(n) e^{n t}+O\left(\varepsilon^{2}\right),
$$

where $f(n)$ is the stability function of (3.17). But $N\left[u_{w}\right]$ is just a function of $u_{w}$ : in fact, from (3.5), $N\left[u_{w}\right]$ is a cubic in $u_{w}$ (see Fig. 2). By Taylor's theorem

$$
N\left[u_{w}(1+\varepsilon)\right]=N\left[u_{w}\right]+\varepsilon u_{w} \frac{d N}{d u_{w}}+O\left(\varepsilon^{2}\right) .
$$

If we put $n=0$ in (3.20), subtract it from (3.21), divide by $\varepsilon$ and let $\varepsilon \rightarrow 0$, we obtain

$$
f(0)=\frac{u_{w}}{\beta(\Delta p)} \frac{d N}{d u_{w}} .
$$

If $N\left[u_{w}\right]$ has a negative slope region $u_{1}<u_{w}<u_{2}$, say, then in this region $d N / d u_{w}<0$, so $f(0)<0$. But as $n \rightarrow+\infty$ along $\operatorname{Im} n=0, f \rightarrow 2 \lambda>0$. Therefore, in $u_{1}<u_{w}<u_{2}$, the system has a static or "Ledinegg" instability (corresponding to a real positive zero of $f(n)$ ). This result was first given nearly 40 years ago (Ledinegg, 1938), but no conclusive proof appears to have been given to date. The converse is also usually taken for granted, that on positive slope regions a static instability cannot exist, but this is not necessarily true, and for sufficiently high inlet subcooling is false for this model, as is shown below by a computed counter example. 


\section{Non-linear Stability}

As we vary the parameters $\mu, \tau_{0}, \lambda$, the zeros of $f(n)$ will vary continuously. Suppose we fix $\mu, \tau_{0}$ and vary only $\lambda$. We know that for $\lambda-\tau_{0} \ll 1$, the system is linearly stable. Therefore, provided the system becomes unstable for $\lambda-\tau_{0}<1$, there will exist a minimum $\lambda, \lambda_{0}$, say, such that the zero of $f(n)$ with greatest real part, $n_{0}$, has $\operatorname{Re} n_{0}=0$. At $\lambda=\lambda_{0}$ the system is said to be in a state of marginal stability. If we increase $\lambda$ slightly further, so $0<\lambda-\lambda_{0} \ll 1$, this root will cross the imaginary axis into the positive half-plane $\operatorname{Re} n>0$. The system is then linearly unstable, but the linear solution becomes invalid for large times since the velocity perturbation, assumed small, tends to infinity. In order to try and obtain an expansion which is valid for all time, we expand the equations about $\lambda_{0}$ and try an asymptotic solution for the velocity of the form

$$
U=1+V, \quad V=\varepsilon V^{(1)}+\varepsilon^{2} V^{(2)} \ldots
$$

where

$$
\varepsilon=\varepsilon(\delta), \quad \delta=\lambda-\lambda_{0}, \quad \varepsilon \rightarrow 0 \text { as } \delta \rightarrow 0 .
$$

Then if $n_{0}=i \Omega$ at $\lambda=\lambda_{0}$, the first-order solution is

$$
V^{(1)}=A_{0} e^{i \Omega t}+\left(^{*}\right)
$$

where $\left({ }^{*}\right)$ denotes the complex conjugate; but, however we choose $\varepsilon(\delta)$, the $O\left(\varepsilon^{3}\right)$ terms include the fundamental $e^{i \Omega t}$, which leads to so-called secular solutions

$$
V^{(3)}=A_{2} t e^{i \Omega t}
$$

as particular integrals. Thus this expansion is also not uniformly valid. However, if we write

$$
V=\varepsilon e^{i \Omega t}\left[A_{0}+\varepsilon^{2} A_{2} t+\ldots\right]+\text { higher harmonics, }
$$

we may suspect that the coefficient of $e^{i \Omega t}$ is the Taylor expansion of a function of the slow time $\varepsilon^{2} t$. Thus we should look for an asymptotic solution

$$
V=\varepsilon A\left(\varepsilon^{2} t\right) e^{i \Omega t}+\ldots
$$

We do this by using the method of multiple scales (Cole, 1968). We write

$$
V=\varepsilon V^{(1)}\left(t^{*}, \tilde{t}\right)+\varepsilon^{2} V^{(2)}\left(t^{*}, \tilde{t}\right)+\ldots, \quad t^{*}=t, \quad \tilde{t}=\varepsilon^{2} t,
$$

with a similar expansion for $\rho$, and formally rewrite the equations (3.10)-(3.13) in terms of $t^{*}$ and $\bar{t}$, expanded about $\lambda_{0}$. Thus, for example, $\partial / \partial t$ is rewritten as $\partial / \partial t^{*}+\varepsilon^{2} \partial / \partial \tilde{t}$. In order to eliminate secular terms, we must be able to equate the coefficients of $e^{i \Omega t^{*}}$ in the $O\left(\varepsilon^{3}\right)$ equation to zero, and for this we must choose

$$
\varepsilon=\delta^{\mathfrak{t}}
$$

because of the appearance of terms of $O(\delta)$ from the expansion of the equations about $\lambda_{0}$. We may solve iteratively for $V^{(r)}$, and we find that the coefficient of $e^{i \Omega t^{*}}$ in the $O\left(\varepsilon^{3}\right)$ equation for $V^{(3)}$ is of the form

$$
m_{1} \frac{d A}{d \tilde{t}}+m_{2} A+m_{3} A|A|^{2}
$$


where $A(\tilde{t})$ is as defined in (4.6) . (4.9) is equated to zero so that the equation for $A(\tilde{t})$ is

$$
\frac{d A}{d \tilde{t}}=k_{1} A+k_{2} A|A|^{2}
$$

where $k_{1}, k_{2}$ are complex constants. The details of this process for the present system are given in Appendix 2 and the values of $k_{1}$ and $k_{2}$ in Appendix 3. One of the main uses of this technique in partial differential equations has been in treating the instability of Poiseuille fiow (Stewartson \& Stuart, 1971). It should be noted that, although that paper considers spatially as well as temporally modulated solutions, there is no need to do so in the present case, since the tube is of finite length.

In (4.10), $\operatorname{Re} k_{1}>0$ if the system is linearly unstable; if $\operatorname{Re} k_{2}<0,|A|$ tends to a finite limit, and there is a small steady oscillatory solution. However, if $\operatorname{Re} k_{2}>0$ then $|A| \rightarrow \infty$ in a finite time, and the solution again becomes invalid. In this case we might expect a large oscillâtion.

\section{Results}

In Fig. 3, a stability máp is shown at a pressure of about 33 bars $(\mu=49)$. The axes are $\tau_{0}$ (a dimensionless measure of the inlet subcooling) and $\lambda-\tau_{0}$ (exit quality): an increase of 0.1 in $\tau_{0}$ corresponds to an increase of about $38.7^{\circ} \mathrm{C}$ in the subcooling at the inlet. The curve marked $N$ denotes values of $\left(\tau_{0}, \lambda-\tau_{0}\right)$ for which the equilibrium pressure drop/flow curve has zero slope, that is, $f(0)=0$. $N$ is simply determined by an examination of the zeros of $d \Delta p / d u_{w}$, as given by (3.5). For large enough $\tau_{0}$, the curve has two branches (the lower being unmarked), and the region where $d \dot{\Delta} p / d u_{w}<0$ lies to the right of the curve: in other regions $d \Delta p / d u_{w}>0$. The curve $L$ divides the plane into a region where there exist Ledinegg instabilities and a region

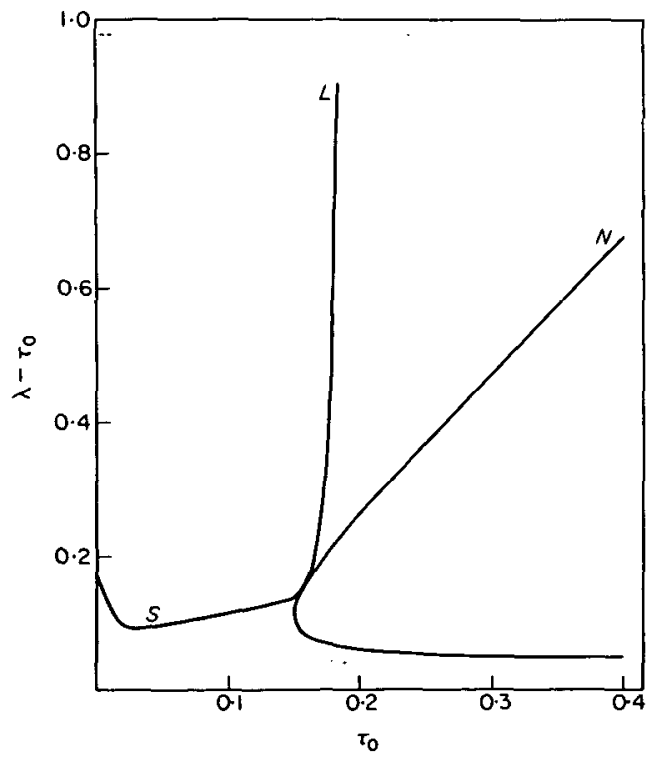

FIG. 3. Linear stability map. 
where there do not (although there may exist oscillatory instabilities). The lower branch of $L$ coincides with that of $N$, and the region of Ledinegg instability lies to the right of $L$. We thus see that there is a large area between the upper branches of $L$ and $N$ in which $d \Delta p / d u_{w}>0$, i.e. $f(0)>0$, but Ledinegg instability exists. This is in disagreement with previous authors. The curve $S$ distinguishes regions of stability from regions of oscillatory instability (it may be called the marginal stability curve). It is considered to terminate when it meets $N$, since it then becomes irrelevant.

To see how the roots of $f(n)$ vary, let us vary $\tau_{0}$ and $\lambda-\tau_{0}$ so that we traverse the stability map in the following way. Let us start at (say) $\left(\tau_{0}, \lambda-\tau_{0}\right)=(0.3,0)$ where the flow is stable. Moving upwards, a real zero crosses $n=0$ from $n<0$ as we cross the lower branch of $N$ (and $L$ ). As we move further upwards and cross the upper branch of $N$, a second zero of $f$ crosses $n=0$ from $n<0$ so that $f(0)>0$ but there are $t w o$ real positive zeros of $f$. As we move sideways towards $L$, these zeros approach each other until, as we cross $L$, they join and bifurcate off the real axis as a conjugate pair of roots in the manner of the roots of a quadratic equation as its discriminant becomes negative. To the left of $L$, the equilibrium is oscillatorily unstable, and as we move downwards to cross $S$, the pair of roots of $f(n)$ with largest real part crosses the imaginary axis into $\operatorname{Re}(n)<0$, so that the steady state is again stable.

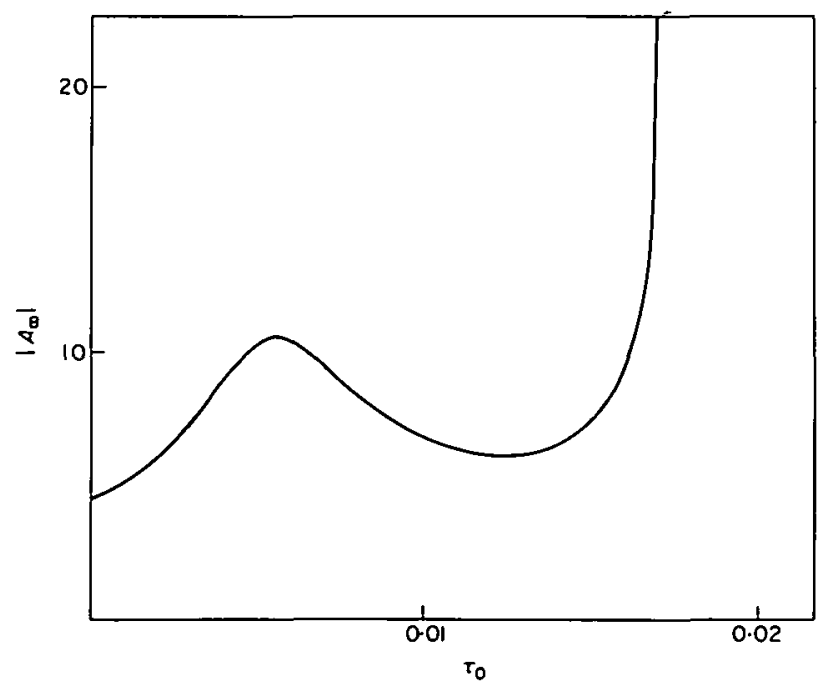

Fig. 4. Variation of limiting amplitude along $S$.

The marginal stability curve marks the transition from stable to oscillatorily unstable flow as $\lambda-\tau_{0}$ increases. For critical $\lambda=\lambda_{0}$, we find that the non-linear analysis predicts a "burst" $\left(|A| \rightarrow \infty\right.$ in (4.10)) if $\tau_{0} \gtrsim 0.017$, otherwise the unstable mode evolves into a small oscillation.

In Fig. 4, a graph of $\left|A_{\infty}\right|$ against $\tau_{0}$ along the marginal stability curve $S$ is shown for $0<\tau_{0} \leqq 0.017$ (note that this is not the same as Fig. 3 of Friedly \& Krishnan (1974), which corresponds to a single point of the present diagram). As $\tau_{0}$ increases, the amplitude of the steady oscillation increases and decreases again before tending to 


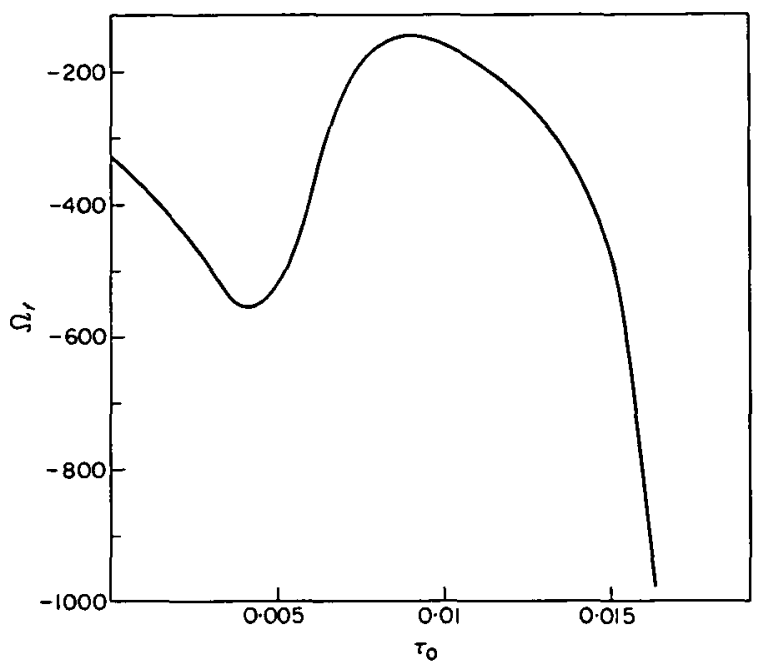

FIG. 5. Variation of limiting frequency along $S$.

infinity as $\tau_{0} \rightarrow \simeq 0.017$. This approach to a "bursting" region may help to explain the dramatic increase in the amplitude of steady oscillations in certain parameter regions measured by Jain (1965). Figure 5 shows the variation of $\Omega_{f}$ as $\tau_{0}$ increases along $S$. $\Omega_{f}$ is the frequency correction defined by

$$
\Omega_{f}=\operatorname{Im} k_{1}+\left|A_{\infty}\right|^{2} \operatorname{Im} k_{2}
$$

using (4.10). The frequency of the limiting oscillation is thus given, from (4.10), by

$$
\Omega_{\infty}=\Omega+\left(\lambda-\lambda_{0}\right) \Omega_{f} .
$$

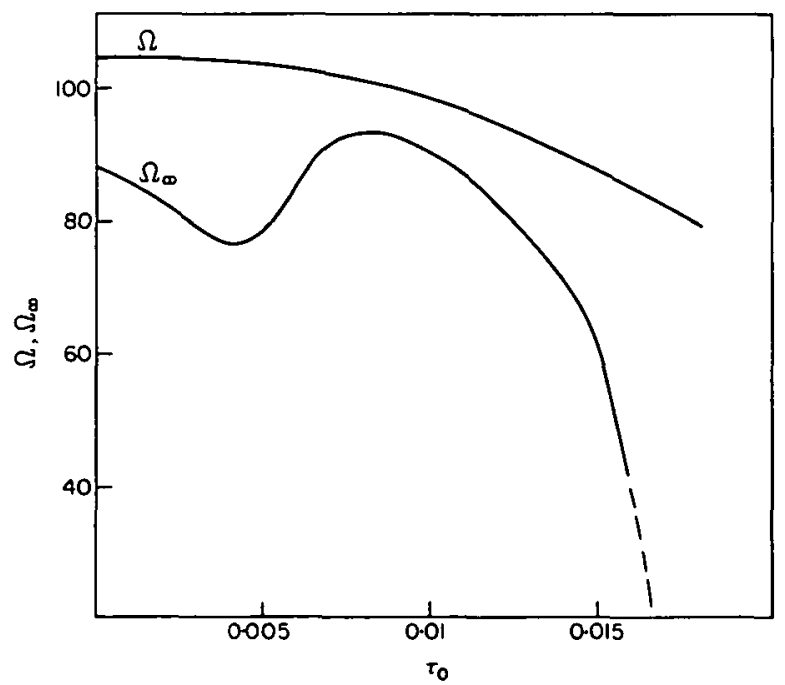

FIG. 6. $\Omega, \Omega_{\infty}$ along $S$ at $\varepsilon^{2}=0.05$. 
It can be seen that $\Omega_{\infty}<\Omega$ in this case and that $\Omega-\Omega_{\infty}$ increases and decreases again before becoming infinite as $\left|A_{\infty}\right| \rightarrow \infty$. An illustrative graph of $\Omega$ and $\Omega_{\infty}$ against $\tau_{0}$ is shown in Fig. 6 for $\varepsilon^{2}=0.05$.

\section{Discussion}

The model proposed above may be expected to exhibit qualitatively similar behaviour to that of actual boilers, and in some cases (where the approximations used are realistic) may be used for predictive purposes as well.

There are two interesting conclusions to be drawn. The first is that Ledinegg instabilities may occur on positive slope regions of the pressure drop/flow curve (and must occur on negative slope regions). The second is that in general if the system has a weak linear instability, then there is an oscillatory solution with limiting amplitude $\left|A_{\infty}\right|$ which varies with the parameters of the problem, but for sufficiently large $\tau_{0}$, the amplitude becomes infinite in this theory. In this case, the asymptotic expansion breaks down, and we should expect that $\left|A_{\infty}\right|$ is in reality finite, but that $\left|A_{\infty}\right| \gg O(\varepsilon)$. As yet, the theory has not been extended to include this case.

Other more obvious conclusions may be drawn from an examination of the stability map, for example the increasing instability as $\lambda$ increases in Fig. 3. In this respect, the results concur with those of other observers.

In conclusion, since this paper may be of interest to practising engineers, I would like to re-emphasise its aim and the importance of the results presented.

The aim has not been to apply the results to any particular model, but rather to show that a qualitative model of the type encountered in heat exchanger dynamics can exhibit both small oscillations and bursts. It is realized that both gravity and inertial terms should be included for any practical application, but it is nevertheless true that an identical analysis to that presented here could still be applied, and the same form of evolution equation (4.10) for small amplitude disturbances would be obtained. The qualitative results obtained here are therefore of interest, and the comparison with Jain's (1965) work is a valid (if not quantitative) one.

From the point of view of the engineer, the result that (for this model) Ledinegg instability may occur on positive slope regions of the equilibrium pressure drop/flow curve is a strange and novel one. It is not due to the pump "operating characteristic" (i.e. $\Delta p$ ) being a function of the inlet velocity : $\Delta p$ is considered to be a constant in this paper. Engineers have always taken it for granted that Ledinegg instability occurs if and only if the equilibrium curve has negative slope $\left(d \Delta p / d u_{w}<0\right)$. This result is simply unproven, and "proofs" like that given by Hands (1975) have to assume that the operator $N$ in (3.19) is an analytic function of $U(t)$, whereas even in the present simple model, such an assumption is obviously unwarranted. Again, R. Potter (private communication, 1975) has commented: “... static instability on a positive sloping characteristic seems impossible ...". On the contrary: it seems impossible to be able to extract information about a complicated dynamical system incorporating (in the present instance) two time delays merely from an examination of its steadystate characteristic. In fact, we have indeed shown that negative slopes of the equilibrium curve imply Ledinegg instability, but this seems to be all that we can usefully say. 
I should like to express my thanks for their helpful advice on this work to Drs A. B. Tayler and J. R. Ockendon of Oxford University, and to the Department of Education of Northern Ireland for financial support while it was being carried out.

\section{REFERENCES}

ColE, J. D. 1968 Perturbation Methods in Applied Mathematics. Waltham, Mass.: Blaisdell. DA vies, A. L. \& PotTer, R. 1967 Hydraulic stability: an analysis of the causes of unstable flow in parallel channels. Paper presented at the Symposium on Two-phase Flow Dynamics, Eindhoven EUR 4288e, 1225-1266.

FriedLy, J. C. \& KrishnaN, V. S. 1974 Predictions of non-linear flow oscillations in boiling channels. AlChE Symp. Ser. 68, 127-135.

HANDS, B. A. 1975 Pressure drop instabilities in cryogenic fluids. Adv. cryo. Engng 20, 355-369.

JAIN, K. C. 1965 Self-sustained hydrodynamic oscillations in a natural circulation two-phase flow boiling loop. ANL-7073.

LEDINEGG, M. 1938 Instability of flow during natural and forced circulation. Die Wärme, 61, 891-898.

Stewartson, K. \& Stuart, J. T. 1971 A non-linear instability theory for a wave system in plane Poiseuille flow. J. Fluid Mech., 48, 529-545.

StuarT, J. T. 1960 On the non-linear mechanics of wave disturbances in stable and unstable parallel flows. J. Fluid Mech. 9, 353-370.

ZUBER, N. 1967 Flow excursions and oscillations in boiling, two-phase flow systems with heat addition. Paper presented at the Symposium on Two-phase Flow Dynamics, Eindhoven EUR 4288e, 1071-1089.

\section{Appendix 1. Linear Stability}

With $U$ and $\rho$ given by (3.15), the equation for $\rho_{0}$ is, on linearizing (3.12),

with

$$
\frac{d \rho_{0}}{d \xi}+(\mu+n) \rho_{0}=\mu\left[1-\frac{1}{n}\left(1-e^{-n \tau_{0}}\right)\right] e^{-2 \mu \xi},
$$

$$
\rho_{0}(0)=\frac{\mu}{n}\left(1-e^{-n \tau_{0}}\right)
$$

The solution is

$$
\rho_{0}=a e^{-(\mu+n) \xi}+b e^{-2 \mu \xi},
$$

where $a$ and $b$ are given by (3.18). The linearized form of (3.13) is

$$
2 \lambda-2 \frac{\mu}{n}\left(\lambda-\tau_{0}\right)\left(1-e^{-n \tau_{0}}\right)+\int_{0}^{\tau_{1}} \rho_{0} e^{3 \mu \xi} d \xi=0 .
$$

Substituting for $\rho_{0}$ from (A1.3), we obtain $f(n)$ as defined in (3.17).

\section{Appendix 2}

We write

$$
\begin{aligned}
t^{*} & =t, \quad \tilde{t}=\varepsilon^{2} t \\
\rho & =e^{-\mu \xi}+\varepsilon \rho^{(1)}\left(\xi, t^{*}, \tilde{t}\right)+\varepsilon^{2} \rho^{(2)}+\ldots, \\
U & =1+\varepsilon V^{(1)}\left(t^{*}, \tilde{t}\right)+\varepsilon^{2} V^{(2)}\left(t^{*}, \tilde{t}\right)+\ldots, \\
\lambda & =\lambda_{0}+\varepsilon^{2}
\end{aligned}
$$


and expand (3.12) and (3.13) in powers of $\varepsilon$. To do this, we use the relations

$$
\begin{gathered}
\partial / \partial t=\partial / \partial t^{*}+\varepsilon^{2} \partial / \partial \tilde{t}, \\
\int_{t-\tau_{0}}^{t} F\left(\dot{\theta}, \dot{\varepsilon}^{2} \theta\right) d \theta=\int_{t^{*}-\tau_{0}}^{t^{*}} F\left(\sigma^{*}, \tilde{t}\right) d \sigma^{*}+\int_{t^{*}-\tau_{0}}^{i^{*}}\left(\sigma^{*}-t^{*}\right) \frac{\partial F}{\partial \tilde{\sigma}}\left(\sigma^{*}, \tilde{t}\right) d \sigma^{*} \ldots,
\end{gathered}
$$

which come from expanding $F\left(\sigma^{*}, \tilde{\sigma}\right)$ about $\tilde{t}$ and using $\tilde{\sigma}-\tilde{t}=\varepsilon^{2}\left(\sigma^{*}-t^{*}\right)$ on the path of integration $\tilde{\sigma}=\varepsilon^{2} \sigma^{*}$ in the $\left(\sigma^{*}, \tilde{\tilde{\sigma}}\right)$ plane.

Equating terms of equivalent order, we find

$$
\begin{aligned}
& O(\varepsilon): \quad L_{11}\left(V^{(1)}\right)+L_{12}\left(\dot{\rho}^{(1)}\right) \equiv L\left(V^{(1)}, \rho^{(1)}\right)=0, \\
& \dot{M}_{11}\left(V^{(1)}\right)+M_{12}\left(\rho^{(1)}\right) \equiv M\left(V^{(1)}, \rho^{(1)}\right)=0, \\
& \rho^{(1)}\left(O, t^{*}, \tilde{t}\right)=B_{1}\left(V^{(1)}\right) ; \\
& O \ddot{\left(\varepsilon^{2}\right):} \quad \dot{L}\left(V^{(2)}, \rho^{(2)}\right)+\dot{L}_{2}\left(V^{(1)}, \rho^{(1)}\right)=0, \\
& M\left(V^{(2)}, \rho^{(2)}\right)+\dot{M}_{2}\left(V^{(1)}, \rho^{(1)}\right)=0, \\
& \rho^{(2)}\left(O, t^{*}, \tilde{t}\right)=B_{1}\left(V^{(2)}\right)+B_{2}\left(V^{(1)}, \rho^{(1)}\right) ;
\end{aligned}
$$

$O\left(\varepsilon^{3}\right): \quad L\left(\dot{V}^{(3)}, \rho^{(3)}\right)+\partial L / \partial \lambda\left(V^{(1)}, \rho^{(1)}\right)+L\left(\dot{V}^{(1)}, \rho^{(1)}\right)+$

$$
\begin{array}{r}
L_{3}\left(V^{(1)}, \rho^{(1)}, V^{(2)}, \rho^{(2)}\right)=0, \\
M\left(V^{(3)}, \rho^{(3)}\right)+M^{\prime}\left(\dot{V}^{(1)}, \rho^{(1)}\right)+M_{3}\left(V^{(1)}, \rho^{(1)}, V^{(2)}, \rho^{(2)}\right)=0, \\
\rho^{(3)}\left(0, t^{*}, \tilde{t}\right)=B_{1}\left(V^{(3)}\right)+B^{\prime}\left(V^{(1)}\right)+B_{3}\left(V^{(1)}, \rho^{(1)}, \dot{V}^{(2)}, \rho^{(2)}\right) .
\end{array}
$$

These operators are defined in Appendix 3:L,M,B$, L^{\prime}, M^{\prime}, B^{\prime}$ and $\partial L / \partial \dot{\lambda}$ are linear $(\partial M / \partial \lambda=0), L_{2}, M_{2}, B_{2}$ are quadratic, and $L_{3}, M_{3}, B_{3}$ are cubic. $\partial L / \partial \lambda$ is the coefficient of $\delta$ in the expansion of $L^{\prime}$ about $\lambda=\lambda_{0}$, and $L^{\prime}, M^{\prime}, B^{\prime}$ arise from the operators involving $\tilde{t}$ in (A2.2) and (A2.3).

The solution of $(A 2.4)$ is

$$
\left(\begin{array}{l}
V^{(1)} \\
\rho^{(1)}
\end{array}\right)=\left(\begin{array}{c}
A(\tilde{t}) e^{i \Omega t^{*}} \\
\rho_{0}(\xi, i \Omega) A(\tilde{t}) e^{i \Omega t^{*}}
\end{array}\right)+\left(^{*}\right),
$$

where $\left(^{*}\right)$ denotes the complex conjugate, and $\rho_{0}$ is defined by (A1.3). We only require a particular solution of (A2.5), since the general solution will not contribute to any secular terms at $O\left(\varepsilon^{3}\right)$.

It is fairly straightforward to show that

$$
\begin{aligned}
\frac{\partial L}{\partial \lambda} & =\frac{\partial f}{\partial \lambda}(i \Omega) A(\tilde{t}) e^{i \Omega t^{*}}, \\
L^{\prime}\left(V^{(1)}, \rho^{(1)}\right) & =\frac{d A}{d \tilde{t}} \frac{\partial f}{\partial n}(i \Omega) e^{i \Omega t^{*}}-\frac{d A}{d \tilde{t}} L\left(0, \frac{\partial \rho_{0}}{\partial n}(\xi, i \Omega) e^{i \Omega \Omega^{*}}\right), \\
M^{\prime}\left(V^{(1)}, \rho^{(1)}\right) & =-\frac{d A}{d \tilde{t}} M\left(0, \frac{\partial \rho_{0}}{\partial n}(\xi, i \Omega) e^{i \Omega 0^{*}}\right),
\end{aligned}
$$

and that$$
\rho^{(3)}=\frac{d A}{d \tilde{t}} \frac{\partial \rho_{0}}{\partial n}(\xi, i \Omega) e^{i \Omega t^{*}}
$$ 
satisfies $\rho^{(3)}\left(0, t^{*}, \bar{t}\right)=B^{\prime}\left(V^{(1)}\right)$. We write

$$
V^{(3)}=u_{1}, \rho^{(3)}=\frac{d A}{d \tilde{t}} \frac{\partial \rho_{0}}{\partial n}(\xi, i \Omega) e^{i \Omega t^{*}}+u_{2},
$$

so that the equations (A2.6) become

$$
\begin{gathered}
L\left(u_{1}, u_{2}\right)+\frac{\partial f}{\partial \lambda} A(\tilde{t}) e^{i \Omega t^{*}}+\frac{d A}{d \tilde{t}} \frac{\partial f}{\partial n} e^{i \Omega t^{*}}+ \\
L_{3}\left(V^{(1)}, \rho^{(1)}, V^{(2)}, \rho^{(2)}\right)=0, \\
M\left(u_{1}, u_{2}\right)+M_{3}\left(V^{(1)}, \rho^{(1)}, V^{(2)}, \rho^{(2)}\right)=0, \\
u_{2}\left(0, t^{*}, \tilde{t}\right)=B_{1}\left(V^{(3)}\right)+B_{3}\left(V^{(1)}, \rho^{(1)}, V^{(2)}, \rho^{(2)}\right) .
\end{gathered}
$$

Finally we solve (A2.11) as a particular integral $u_{1}=u_{1 p}, u_{2}=u_{2 p}$ so that the non-linear part of the boundary condition (A2.12) is satisfied; then, with $\theta_{1}=u_{1}-u_{1 p}, \theta_{2}=u_{2}-u_{2 p}$, we have

$$
\begin{aligned}
L\left(\theta_{1}, \theta_{2}\right)+\left[\frac{\partial f}{\partial \lambda} A(\tilde{t})+\frac{d A}{d \tilde{t}}\right. & \left.\frac{\partial f}{\partial n}+m_{3} A|A|^{2}\right] e^{i \Omega t^{*}}+\left(^{*}\right) \\
& =\text { higher harmonics, } \\
M\left(\theta_{1}, \theta_{2}\right) & =0, \\
\theta_{2}\left(0, t^{*}, \tilde{t}\right) & =B_{1}\left(\theta_{1}\right),
\end{aligned}
$$

where $m_{3} A|A|^{2}$ is the coefficient of $e^{i \Omega t^{*}}$ in

$$
L_{3}\left(V^{(1)}, \rho^{(1)}, V^{(2)}, \rho^{(2)}\right)+L\left(u_{1 p}, u_{2 p}\right),
$$

$m_{3}$ being a function of $\Omega$. The condition that the solution of (A2.13) should contain no secular terms is that

$$
\frac{\partial f}{\partial n} \frac{d A}{d \tilde{t}}+\frac{\partial f}{\partial \lambda} A+m_{3} A|A|^{2}=0
$$

or

$$
\frac{d A}{d \tilde{t}}=k_{1} A+k_{2} A|A|^{2}
$$

where

is the linear growth rate,

$$
k_{1}=\frac{d n_{0}}{d \lambda}\left(\lambda_{0}\right)
$$

$$
k_{2}=-m_{3} / \frac{\partial f}{\partial n}
$$

\section{Appendix 3}

All indefinite integrals in this section are between $t^{*}-\tau_{0}$ and $t^{*}$, and $\rho(0)$ is an abbreviation for $\rho\left(0, t^{*}, \tilde{t}\right)$. The operators and functions referred to in Appendix 2 are given by 


$$
\begin{gathered}
B_{1}=\mu \int V^{(1)} \\
B_{2}=-\left[\rho_{\xi}^{(1)}(0) \int V^{(1)}+\mu^{2} \int^{2} V^{(1)}\right]
\end{gathered}
$$

in (A3.2), the second term is an abbreviation of $\left(\int V^{(1)}\right)^{2}$, and similar notation will be employed subsequently.

$$
\begin{gathered}
B_{3}=-\left[\rho_{\xi}^{(1)}(0) \int V^{(2)}+\rho_{\xi}^{(2)}(0) \int V^{(1)}+\frac{1}{2} \int^{2} V^{(1)} \times\right. \\
\left.\left\{\rho_{\xi \xi}^{(1)}(0)-\mu \rho_{\xi}^{(1)}(0)\right\}+2 \mu^{2} \int V^{(1)} \int V^{(2)}-\mu^{3} \int^{3} V^{(1)}\right] \\
b=\frac{\mu}{n-\mu}\left[1-\frac{\mu}{n}\left(1-e^{\left.-n \tau_{0}\right)}\right], a=\frac{\mu}{n}\left(1-e^{-n \tau_{0}}\right)-b\right. \\
\rho_{0}=a e^{-(n+\mu) \xi}+b e^{-2 \mu \xi}, \\
M_{11}(V)=-\mu\left[V-\mu \int V\right] e^{-2 \mu \xi}, \\
M_{12}(\rho)=\rho_{r^{*}}+\rho_{\xi}+\mu \rho, \\
2\left\{V^{(1)}-\mu \int V^{(1)}\right\} \int_{0}^{\tau_{1}} \rho^{(1)} e^{2 \mu \xi} d \xi+\frac{1}{2} \mu \int^{2} V^{(1)}, \\
M_{2}\left(V^{(1)}, \rho^{(1)}\right)=e^{-\mu \xi} \rho_{\xi}^{(1)}\left[V^{(1)}-\mu\left[V^{(1)}\right]\right. \\
L_{11}^{(1)}(V)=2 \lambda V-2 \mu\left(\lambda-\tau_{0}^{(1)}\right)=\tau_{0} V^{(1)^{2}}+\tau_{1}\left\{V^{(1)}-\mu \int V^{(1)}\right\}^{2}+e^{\tau_{1}} \rho e^{3 \mu \xi} d \xi \\
M_{3}\left(V^{(1)}, \rho^{(1)}, V^{(2)}, \rho^{(2)}\right)=e^{-\mu \xi} \rho_{\xi}^{(1)}\left[V^{(2)}-\mu \int V^{(2)}\right]+
\end{gathered}
$$




$$
\begin{aligned}
& L_{3}\left(V^{(1)}, \rho^{(1)}, V^{(2)}, \rho^{(2)}\right)=2 \tau_{0} V^{(1)} V^{(2)}+\frac{1}{2} \rho_{\xi}^{(1)}(0) \int^{2} V^{(1)}+\mu^{2} \int^{3} V^{(1)}+ \\
& \mu \int V^{(1)} \int V^{(2)}+2 \tau_{1}\left\{V^{(1)}-\mu \int V^{(1)}\right\} \times \\
&\left\{V^{(2)}-\mu \int V^{(2)}\right\}+\left\{V^{(1)}-\mu \int V^{(1)}\right\}^{2} \times \\
& \int_{0}^{\tau_{1}} \rho^{(1)} e^{\mu \xi} d \xi+2\left\{V^{(2)}-\mu \int V^{(2)}\right\} \times \\
& \int_{0}^{\tau_{1}} \rho^{(1)} e^{2 \mu \xi} d \xi+2\left\{V^{(1)}-\mu \int V^{(1)}\right\} \times \\
& \int_{0}^{\tau_{1}} \rho^{(2)} e^{2 \mu \xi} d \xi .
\end{aligned}
$$

(A3.12) and (A3.13) have been simplified by using (A3.1) and (A3.2) in the values for $\rho^{(1)}(0)$ and $\rho^{(2)}(0)$.

By inspection of (A3.6), (A3.7), (A3.8), the particular solution of (A2.5) is

$$
\begin{aligned}
& V^{(2)}=\alpha_{0}|A|^{2}+\alpha_{2} e^{2 i \Omega i^{*}}+\left(^{*}\right), \\
& \rho^{(2)}=|A|^{2}\left[c_{0} e^{-\mu \xi}+c_{1} e^{-2 \mu \xi}+c_{2} e^{-(i \Omega+2 \mu) \xi}+c_{3} e^{-3 \mu \xi}\right]+A^{2} e^{2 i \Omega i^{*}} \times \\
& \quad\left[d_{0} e^{-(\mu+2 i \Omega) \xi}+d_{1} e^{-2 \mu \xi}+d_{2} e^{-(i \Omega+2 \mu) \xi}+d_{3} e^{-3 \mu \xi}\right]+\left(^{*}\right) .
\end{aligned}
$$

Substituting (A3.14) into (A2.5), we obtain

$$
\begin{gathered}
c_{3}=-b U(-i \Omega), \\
c_{2}=-a U(-i \Omega), \\
d_{2}=(i \Omega+\mu) a b / \mu, \\
d_{3}=b^{2}, \\
\alpha_{0}=\frac{-\left[\frac{1}{2} \mu R(i \Omega) R(-i \Omega)+\tau_{0}+\tau_{1} U(i \Omega) U(-i \Omega)+2 U(-i \Omega) R 2+c_{3} \tau_{1}+\right.}{\left.c_{2} D(\mu-i \Omega)+D(2 \mu)\left\{R(-i \Omega) R 3-c_{2}-c_{3}\right\}\right]} \\
\alpha_{2}=\frac{c_{1}=\alpha_{0}\left(\mu \tau_{0}-1\right),}{\left[2 \mu \tau_{0}^{2}+\left(\mu \tau_{0}-1\right)\left\{D(\mu)-D(2 \mu)-2 \lambda_{0}\right\}\right]} \\
c_{0}=R(-i \Omega) R 3-\left(c_{1}+c_{2}+c_{3}\right), \\
\left.d_{3} \tau_{1}+\tau_{0}+\tau_{1} U^{2}(i \Omega)+2 U(i \Omega) R 2+\frac{1}{2} \mu R^{2}(i \Omega)\right]
\end{gathered}
$$


where

$$
\begin{aligned}
R(z) & =\frac{1}{z}\left(1-e^{-z \tau_{0}}\right), \\
D(z) & =\int_{0}^{\tau_{1}} e^{z \xi} d \xi=\frac{1}{z}\left(e^{z \tau_{1}}-1\right), \\
U(z) & =1-\mu R(z) \\
V(z) & =\frac{U(z)}{z-\mu} \\
R 3 & =(i \Omega+\mu) a+2 \mu b-\mu^{2} R(i \Omega), \\
R 2 & =a D(\mu-i \Omega)+b \tau_{1},
\end{aligned}
$$

and $a, b$ are given by (A3.4).

Substituting (A3.14) and (A2.7) into (A3.9), we find that a particular integral of (A2.11) satisfying the boundary condition $\rho^{(3)}(0)=B_{3}$ (given by (A3.3)) is

$$
\begin{aligned}
& V^{(3)}=0, \\
& \rho^{(3)}=|A|^{2} A e^{i \Omega i^{*}}\left[f_{0} e^{-(2 \mu+i \Omega) \xi}+f_{1} e^{-3 \mu \xi}+f_{2} e^{-(2 \mu-i \Omega) \xi}+\right. \\
& \\
& f_{3} e^{-2 \mu \xi}+f_{4} e^{-4 \mu \xi}+f_{5} e^{-(i \Omega+3 \mu) \xi}+f_{6} e^{-(3 \mu-i \Omega) \xi}+ \\
& \left.f_{7} e^{-(2 \mu+2 i \Omega) \xi}+f_{8} e^{-(\mu+i \Omega) \xi}\right],
\end{aligned}
$$

where the $f_{i}$ are given by

$$
\begin{aligned}
& f_{0}=-(\mu+i \Omega) \frac{a}{\mu}\left(\alpha_{0}+\bar{\alpha}_{0}\right)\left(1-\mu \tau_{0}\right) \\
& f_{1}=\frac{2 \mu}{(i \Omega-2 \mu)}\left[b\left(\alpha_{0}+\bar{\alpha}_{0}\right)\left(1-\mu \tau_{0}\right)+\bar{b} \alpha_{2} U(2 i \Omega)+\right. \\
& f_{2}=(\mu-i \Omega) \bar{a} \alpha_{2} V(2 i \Omega), \\
& f_{3}=\mu\left(c_{0}+\bar{c}_{0}\right) V(i \Omega) \\
& f_{4}=\frac{3 \mu}{(i \Omega-3 \mu)}\left[\left(c_{3}+\bar{c}_{3}\right) U(i \Omega)+d_{3} U(-i \Omega)\right] \\
& f_{5}=\frac{-(2 \mu+i \Omega)}{2 \mu}\left[c_{2} U(i \Omega)+d_{2} U(-i \Omega)\right] \\
& f_{6}=\frac{1}{2} \bar{c}_{2}(2 \mu-i \Omega) V(i \Omega) \\
& f_{7}=(\mu+2 i \Omega) d_{0} V(-i \Omega) \\
& f_{8}=-\left(f_{1}+f_{2}+f_{3}+f_{4}+f_{5}+f_{6}+f_{7}\right)+\Sigma
\end{aligned}
$$

Here a bar denotes the complex conjugate, and $\Sigma$ is the coefficient of $|A|^{2} A e^{i \Omega t^{*}}$ in (A3.3), given by

$$
\begin{aligned}
\Sigma= & L 2\left[R 4-2 \mu^{2} R(i \Omega), \alpha_{0} \tau_{0}, \alpha_{2} R(2 i \Omega)\right]+ \\
& L 2\left[R(i \Omega), \mu c_{0}+2 \mu c_{1}+(i \Omega+2 \mu) c_{2}+3 \mu c_{3},\right. \\
& \left.(\mu+2 i \Omega) d_{0}+2 \mu d_{1}+(i \Omega+2 \mu) d_{2}+3 \mu d_{3}\right]+ \\
& L 3\left[R(i \Omega), R(i \Omega), \mu^{3} R(i \Omega)-\frac{1}{2} \mu R 4-\frac{1}{2}(i \Omega+\mu)^{2} a-2 \mu^{2} b\right],
\end{aligned}
$$


where

$$
\begin{aligned}
R 4 & =(i \Omega+\mu) a+2 \mu b, \\
L 2\left[z_{1}, z_{2}, z_{3}\right] & =z_{1}\left(z_{2}+\bar{z}_{2}\right)+\bar{z}_{1} z_{3}, \\
L 3\left[z_{1}, z_{2}, z_{3}\right] & =z_{1} z_{2} \bar{z}_{3}+z_{1} \bar{z}_{2} z_{3}+\bar{z}_{1} z_{2} z_{3} .
\end{aligned}
$$

$L 2$ and $L 3$ give the coefficients of $|A|^{2} A e^{i \Omega t^{*}}$ in products of (i) an $O(\varepsilon)$ and $O\left(\varepsilon^{2}\right)$ term, (ii) three $O(\varepsilon)$ terms, respectively.

Thus the particular solutions $u_{1 p}=0, u_{2 p}$ are given by (A3.26), and

$$
\begin{aligned}
L\left(u_{1 p}, u_{2 p}\right) & =L_{12}\left(u_{2 p}\right)=\int_{0}^{\tau_{1}} \dot{u}_{2 p} e^{3 \mu \xi} d \xi \\
& =f_{0} D(\mu-i \Omega)+f_{1} \tau_{1}+f_{2} D(\mu+i \Omega)+f_{3} D(\mu)+f_{4} D(-\mu)+ \\
& \quad f_{5} D(-i \Omega)+f_{6} D(i \Omega)+f_{7} D(\mu-2 i \Omega)+f_{8} D(2 \mu-i \Omega) \\
& =Q_{1}, \text { say. }
\end{aligned}
$$

It remains to compute the coefficient of $|A|^{2} A e^{i \Omega t^{*}}$ in $L_{3}$ (A3.13). This is

$$
\begin{aligned}
Q_{2}= & 2 \tau_{0} L 2\left[1, \alpha_{0}, \alpha_{2}\right]+\mu L 2\left[R(i \Omega), \alpha_{0} \tau_{0}, \alpha_{2} R(2 i \Omega)\right]+ \\
& 2 L 2\left[R 2, \alpha_{0}\left(1-\mu \tau_{0}\right), \alpha_{2} U(2 i \Omega)\right]+ \\
& L 3\left[R(i \Omega), R(i \Omega), \mu^{2} R(i \Omega)-\frac{1}{2} R 4\right]+ \\
& 2 L 2\left[U(i \Omega), \tau_{1} \alpha_{0}\left(1-\mu \tau_{0}\right)+c_{0} D(\mu)+c_{1} \tau_{1}+c_{2} D(-i \Omega)+c_{3} D(-\mu),\right. \\
& \left.\quad \tau_{1} \alpha_{2} U(2 i \Omega)+d_{0} D(\mu-2 i \Omega)+d_{1} \tau_{1}+d_{2} D(-i \Omega)+d_{3} D(-\mu)\right]+ \\
& L 3[U(2 i \Omega), U(2 i \Omega), a D(-i \Omega)+b D(-\mu)] .
\end{aligned}
$$

Thus, $m_{3}$ in equation (4.9) is given by

$$
m_{3}=Q_{1}+Q_{2} .
$$

$\partial f / \partial n, \partial f / \partial \lambda$ may be found explicitly simply by differentiating $f(n)$. 